\title{
A New Trade marketing Technology:Panoramic Demonstration Marketing of Products
}

\author{
Zebang Tao ${ }^{1, *}$, Xuebin Deng ${ }^{2, *}$, Hong Chen ${ }^{3, *}$, Fenglan Wang ${ }^{4, *}$ and Xiaohui \\ $\mathrm{Hou}^{5, *}$
}

${ }^{1}$ Guangzhou Huali Science and Technology Vocational College ,Guangzhou 510000, China;

${ }^{2}$ Guangzhou branch of Chinese mercantile bank,Guangzhou 510000, China;

${ }^{3}$ GF securities Co. ,Ltd,Guangzhou 510000, China;

${ }^{4}$ Guangdong College of Business and Technology,Zhaoqing 526000, China;

${ }^{5}$ Guangzhou Yuanhong supply-chain management Co., Ltd,Guangzhou 510000, China.

*Corresponding author Email:527443428@qq.com

\begin{abstract}
Key words: Panoramic demonstration marketing of products,factor, value of the product,technology type
\end{abstract}

\begin{abstract}
Panoramic demonstrate marketing of products is a new trade marketing technology.Panoramic demonstrate marketing has a high commercial value and good marketing effect.For this reason,this kind of trade marketing strategy is useful for enterprises.By the way,panoramic demonstrate marketing of products is also a new direction of marketing theory research, business practice and marketing teaching.This paper aims to puts forward a complete definition of the products' panoramic demonstrate marketing through the observation,analysis and arrangement of the demonstrate marketing of products. In this paper,we shall research and analyze the component elements of the panoramic demonstrate marketing of products and the value of the products which have been presented through the panoramic demonstrate marketing.Thereby, we put forward some specific and operable technology types and provide it to mass marketing research scholars and enterprises as a reference.Therefore,this paper has a high practical value and social significance.
\end{abstract}

\section{New trade marketing mode:Panoramic Demonstration Marketing of Products}

A Panoramic Demonstration Marketing of Products,as the name suggests,is to make a all-round, integrity demonstration and deduction of the products.At the same time, transfer the characteristics and advantages of the products to the customer effectively and perfect.Thus make customers produce a series of actions from being attracted ,deepen understanding to order the products excited.Finally achieve the results of marketing successful.Actually, Panoramic Demonstrate Marketing of Products is a new field,the theoretical research about it is relatively few.Maybe business practice will be a little bit more,but still relatively scattered, not a system. The demonstrate marketing of products that we can see nowadays is just PPT demo, video presentation, or super-add the contents of personal presentation.,In essence, these presentations are just some parts or certain angles of the panoramic demonstrate marketing of products. They are not complete.From the influence,propagation degree and business results, throughout the vast majority of business applications of demonstrate marketing,it can be said that only the new product launch of Jobs which from Apple Corp was truly a panoramic demonstrate marketing of products.

Each time,when Jobs make a panoramic demonstrate marketing of the new products ,it will be 
have a lively and shocking atmosphere.So that people could completely, authentic taste of the charm of the target product.The whole marketing process can be summed up with three words, " lively, vivid, active".After finished the demonstrate marketing of the products, it will set off a wave of buying Apple Corp products immediately.We can often see long queues of panic buying on the news,then it will have a great influence on the world and a rapid growth in sales of the target products.Now, more and more enterprises have began to learn the Jobs' demonstrate marketing model of press conference for new products.

\section{Factors of Panoramic demonstration marketing of products}

Jobs' press conference for new products is the example of Panoramic demonstrate marketing so far. According to our observation, the panoramic demonstrate marketing of products at least includes these three ways of demonstration. The formula is as follows:

Panoramic demonstration of products $=$ character demonstration + screen demonstration + entity demonstration

Character demonstration, is to demonstrate the products through the the character's language, behavior, and play .Including personal speech, partner cooperation and teamwork and so on.

Screen demonstration, is to demonstrate the products through text, pictures, videos on the screen, including PPT presentations, picture presentations and video presentations and so on.

entity demonstration, that is, through the ways of display, operate, testing and other products to demonstrate the products , including the physical display, physical operations, physical testing and so on.

These above three ways,is a combination of our traditional ways of demonstration.They are more effective and more comprehensive than the simple personal speech, PPT demonstration and video demonstration to present the value of the target product.

Of course, except the three main presentation, there may be some other auxiliary demonstration way which not included in the formula. With the times and social development, the emergence of some new demonstration mode may also be appear in the future .Therefore, strictly speaking, the formula of panoramic demonstration of products should be modified as follows:

Panoramic demonstration of the products $=$ character demonstration + screen demonstration + real demonstration + other presentations

\section{The product value presented by Panoramic Demonstration Marketing of Products}

The most important of panoramic demonstration marketing of products is to present the value of the target product. In accordance with the actual situation, the value of the products include the quality, quantity, weight, function, price, specifications, features, simplicity, concessions and servicesDifferent enterprises have different product value, generally speaking, it can be divided into three categories, the formula is as follows:

Product value $=$ direct value + added value + Meaning value

Direct value, such as quality, quantity, weight, function, price, specification, etc. Which belongs to the material level of value and most concerned about,needed by consumers.

Added value, such as specifications terms,concessional terms, after sales service, gift giving, and so on. Which belongs to the material level of value and is relative cared and indirect needed by consumers.

Meaning value, such as the well-being , peace, health, happiness, sweet, elegant, high-end and exquisite that has been taken by the target product.These have a certain degree of belonging to the spiritual level of value.

The value that appears must be closely around "what are the benefits to customers, and the benefits of the target product are better than those of other companies and other products ".This is the value of our own company's products that take to the customer. 


\section{Different modes of Panoramic demonstration marketing of products}

All Above are the research and analysis of the panoramic demonstration marketing of products.Specifically speaking,how to use panoramic demonstration marketing of products to commercial practice or enterprise actual combat to achieve the effects of" lively, vivid, active".According to the observation about panoramic demonstration marketing of the products , you can have the following practices:

4.1comparative demonstration:cause a strong shock with the power of contrast.comparative demonstration is to give customers a strong contrast through the gap between the target product and other products ,thus highlight the advantages of the target product.

4.1.1Compared with similar products.If the contrast of the product is not the same product, then it could not called a comparative demonstration.Only when compared with similar products, can display the huge difference.For example, we want to use a comparative demonstration to demonstrate the car.We should use our own brand cars to compare with other brands of cars, and then highlight the advantages of our own brand cars. This is the contrast between the car brands. Or we can use our own brand of cars to compare with motorcycles, bicycles, and even the carriage.And then highlight the advantages of our own brand cars,that is the comparison between transport.

4.1.2 Compare their situations to our past.We are compare with other people just now,we can also compare their situations to our past.Use the current brands to compare with the bygone brands,so as to highlight the areas which have been improved, innovated..It can also show the advantages of our own current brands.For example, the cars of our current brand are more comfortable, faster and more fuel-efficient. While the cars of bygone brand run slowly has a narrow space and high fuel consumption.Compared with the the old one,the new one has more advantages.

4.1.3 The contrast is bigger,the better.When using the comparative demonstration,we should take the advantages of the target products to compare with the disadvantages of the comparative products,thereby highlight target products' strengths.If the gap between the advantages and disadvantages of the contrast is greater,than the customers can feel more deeply.As a result,we can more able to show the advantages of the target product.Therefore,it could be much better if the contrast is bigger.

4.2.Setting off demonstration; Although the flower is beautiful, it is also necessary to set off by the green leaves. Setting off demonstration is to take customers into a beautiful, attractive picture, through the auxiliary products against the target product.Thereby highlight the advantages of the target products.

4.2.1Although the flower is beautiful, it is also necessary to set off by the green leaves. Set off demonstration is to take customers into a beautiful, attractive picture, through the auxiliary products against the target product .By the setting off of the auxiliary products.Setting off demonstration is different from the comparative demonstration.Comparative demonstration is to highlight the advantages of the target products through the contrast of the similar products.For example, we demonstrate the target products: Delicious instant noodles. Then we can add flexible sausage,fragrant smell , boiling water and gluttonous fat boy into a group.All of these can serve to highlight the color and aroma of the target product.

4.2.2 Wonderful auxiliary products.We should set off the advantages of the target products through the wonderful auxiliary products,rather than through the defect of the auxiliary products .For example,we would like to use flexible sausage,fragrant smell , boiling water and gluttonous fat boy to set off the Delicious instant noodles.But not skinny dry sausage,cold water and an ordinary passer-by.

4.3.Sharing demonstration:It's really good when everybody is good. Sharing demonstration is to invite customers to trial products or share feelings,so as to increase the product experience and sense of identity of the customer group.

4.3.1 It's not what we think highly of the products, what matters most is how the customers feel about the products. When we make the products demonstration . 
When we are making product demonstration, we may tell other people that the target product is very good since it's very convenient to use.And we fell joyful and pleased when we using this kind of products.But don't forget that we are thrasonical when we do all this things.At this time,if we Share the product on demonstration stage, we can get a lot of benefits.First,it can be more persuasive because the customers also think highly of your products.Second,customers can feel much more deeper if they can trail this kind of products.

4.3.2 To guide the customers sharing their feelings.If the customers try the products,they may have some profound feelings.The customers may don't know take the initiative to express this feeling.On the other hand, if customers want to take the initiative to express this feeling,but he may not know how to express and praise appropriate.At this time, the presenter should guide the customer to share their feelings,step by step,as we often see the TV host guide the guests and the audience through induction.

4.4.Experimental demonstration:Speak with truth because the truth speaks louder than words.Experimental demonstration is to operate and test the products.And allow customers to see the effect of the product so as to increase the product's persuasion.

4.4.1 Do a actual experiment.The presenter's presentation is his boast. while the Sharing demonstration boasted by other people. Experimental demonstration is to speak with the facts.It will be much better if we do a experiment than just say a lot of superfluous words.

4.4.2The experiment should be progressive.Each experiment should more difficult than the previous one, and the results of each experiment are more convincing than the previous one.

For example, we conduct an experiment which use hammer to knock a glass to prove the robustness of the glass products.

First time, we can seek a female client to knock. Second time, we can seek a male customer to knock. While at the third time,we can ask a strong male customers, or even invite a fitness trainer, athlete, Hercules to knock.There is no doubt that it will be much more persuasive than only to find a regular customer to knock the glass.

4.5.Story demonstration:Tell the story behind the products.Story demonstration,,is to highlight the advantages of products by the way of the story.

4.5.1There should be some difficulties in the story.We can't impressive

the customers if we demonstrate an ordinary story.So,there should be some difficulties when setting the story.And because of this kind of products,we can solve this problems easily.

4.5.2 There should be ups and downs in the story.It's not enough as there are difficulties in the story. If this is a difficult problem is easy to solve,then the story will become a simple account.The difficulty in the story should be powerful,it could not be overcome although we struggle for it hardly.Finally, with the help of the target products,miracle appears.

4.6.Significance demonstration:The significance is more important than the products.

Significance demonstration is to let the customers feel the significance of the products through the perfect and ideal state of the target product.For example,actually,we are demonstrating a family when we demonstrating a house.Family, is the value of the house.We demonstrate sports equipment, through the use of equipment after the effect of the motion picture, such as ruddy face, strong body, to demonstrate health.Health, is the significance of sports equipment. When we demonstrate clothing accessories,we are demonstrating the quality of life at the same time.Therefore,Panoramic Demonstration Marketing of Products aims to let the customers get to know that we are not only selling products, we also sell dreams, the way of life, life attitude and taste of life.

\section{Summary:}

Panoramic Demonstration Marketing of Products start from Jobs' press conference for new products and it was copied by more and more enterprise.Nowadays,it applied in investment road shows, real estate development business, the company opened, the company reception, and even a large number of enterprises in the exhibition, especially in the Canton Fair to do product exhibition.With the further development of the economy, we believes that Panoramic 
Demonstration Marketing of Products will be applied in more and more areas, and further lead the trend of marketing.

\section{Acknowledgments}

The first, second, third, fourth, fifth authors are Zebang Tao,Xuebin Deng, Hong Chen, Fenglan Wang and Xiaohui Hou.The co corresponding authors are Zebang Tao,Xuebin Deng, Hong Chen, Fenglan Wang and Xiaohui Hou.

Supported by an innovation and entrepreneurship training project of university students which named "Independent College 'business presentation' second class training" (item No. 1365612047),is one of an annual"Quality Project"in Colleges and Universities of Guangdong Province in 2012.This paper also won the second prize of Guangdong provincial association's outstanding achievements in 2016 .The relevant equipment, 360 degree full range simulator,has obtained practical new-type patent of State Intellectual Property Office(Patent No.: ZL201520833579.X).

\section{References:}

[1]. Song Yan, Shi Liping curriculum reform based on "business planning and presentation" TOPCARES-CDIO model [J] Vocational Education Research, 2012 (10): 100-101.

[2].Wang Kun. Research on dynamic business presentation system of Lingo language (J). Information technology, 2013 (9): 117-120.

[3].. Yang Wancheng, Tongxia Min The 3D Demonstration Research of B2C enterprise products in Electronic Commerce [J] Chinese commerce, 2011. (9): 115-116.

[4].Xiao Hui. Make business presentations more hommization(J). Computer knowledge and technology. 2013 (9): 99-100.

[5]. Lili Liao ,Huifang Li , Yuanfeng Yang ,Minya Zhao . Application Research of electric commerce practical teaching platform based on demonstration and case (J). Computer knowledge and technology. 2010 (27): 97-98. 